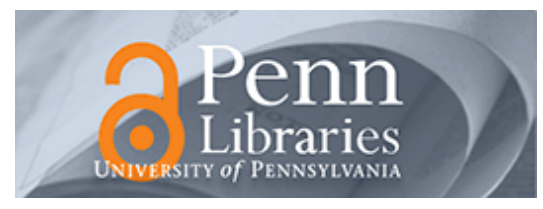

University of Pennsylvania ScholarlyCommons

$12-2012$

\title{
Duopoly Pricing Game in Networks With Local Coordination Effects
}

Arastoo Fazeli

University of Pennsylvania

Ali Jadbabaie

University of Pennsylvania, jadbabai@seas.upenn.edu

Follow this and additional works at: https://repository.upenn.edu/ese_papers

Part of the Controls and Control Theory Commons, Dynamic Systems Commons, and the Other Applied Mathematics Commons

\section{Recommended Citation}

Arastoo Fazeli and Ali Jadbabaie, "Duopoly Pricing Game in Networks With Local Coordination Effects", . December 2012.

Fazeli, A. \& Jadbabaie, A. (2012). Duopoly Pricing Game in Networks With Local Coordination Effects. Proceedings of the 51st IEEE Conference on Decision and Control, Maui, Hawaii, pp. 2684-2689.

(C) 2012 IEEE. Personal use of this material is permitted. Permission from IEEE must be obtained for all other uses, in any current or future media, including reprinting/republishing this material for advertising or promotional purposes, creating new collective works, for resale or redistribution to servers or lists, or reuse of any copyrighted component of this work in other works.

This paper is posted at ScholarlyCommons. https://repository.upenn.edu/ese_papers/619

For more information, please contact repository@pobox.upenn.edu. 


\title{
Duopoly Pricing Game in Networks With Local Coordination Effects
}

\begin{abstract}
In this paper, we study a duopoly pricing problem in which two firms compete for selling two products in a network. Our proposed model consists of two stages. In the first stage, firms set the price they charge agents for their product and the quality of the product they offer. For agents, the quality of the product can be interpreted as the payoff of a local coordination game played among them in the network. In the second stage, agents in the network decide what fraction of these two products to purchase. We first characterize the Nash equilibrium of the game played among agents in the network. We show that agents' actions in the Nash equilibrium consist of two terms, one of which is proportional to the agents' centrality in the network. Conditioned on agents playing the equilibrium policy, we find the Nash equilibrium of the pricing game played between firms. We show that even when firms are similar and offer a uniform price for agents, their Nash equilibrium price depends on the network structure. We then analyze sensitivity of the agents' consumption with respect to the price and quality of the product. We finally show that depending on a firm's opponent's price and quality, the optimal price of a firm can be higher, equal or less than the monopoly optimal price.
\end{abstract}

\section{Keywords}

Game Theory, Social Networks, Pricing

Disciplines

Controls and Control Theory | Dynamic Systems | Other Applied Mathematics

\section{Comments}

Fazeli, A. \& Jadbabaie, A. (2012). Duopoly Pricing Game in Networks With Local Coordination Effects. Proceedings of the 51st IEEE Conference on Decision and Control, Maui, Hawaii, pp. 2684-2689.

(C) 2012 IEEE. Personal use of this material is permitted. Permission from IEEE must be obtained for all other uses, in any current or future media, including reprinting/republishing this material for advertising or promotional purposes, creating new collective works, for resale or redistribution to servers or lists, or reuse of any copyrighted component of this work in other works. 


\title{
Duopoly Pricing Game in Networks With Local Coordination Effects
}

\author{
Arastoo Fazeli $^{\dagger} \quad$ Ali Jadbabaie $^{\dagger}$
}

\begin{abstract}
In this paper, we study a duopoly pricing problem in which two firms compete for selling two products in a network. Our proposed model consists of two stages. In the first stage, firms set the price they charge agents for their product and the quality of the product they offer. For agents, the quality of the product can be interpreted as the payoff of a local coordination game played among them in the network. In the second stage, agents in the network decide what fraction of these two products to purchase. We first characterize the Nash equilibrium of the game played among agents in the network. We show that agents' actions in the Nash equilibrium consist of two terms, one of which is proportional to the agents' centrality in the network. Conditioned on agents playing the equilibrium policy, we find the Nash equilibrium of the pricing game played between firms. We show that even when firms are similar and offer a uniform price for agents, their Nash equilibrium price depends on the network structure. We then analyze sensitivity of the agents' consumption with respect to the price and quality of the product. We finally show that depending on a firm's opponent's price and quality, the optimal price of a firm can be higher, equal or less than the monopoly optimal price.
\end{abstract}

\section{INTRODUCTION}

Many recent studies have documented the role of social networks in individual purchasing decisions [1]-[3]. As a result, retail firms have become more interested in utilizing research on social networks in order to improve their business strategies. In particular, considering the relationship between people in social networks and their rational choices, many retailers are interested to know how to optimize their business decisions in a competitive market. It is therefore important for firms to understand how to shape their strategies in pricing of their products in order to achieve the most profit in selling their products in social networks.

To this end, we study a duopoly pricing problem in which two firms compete for selling two substitute products in a network. A substitute is a product or service that satisfies the need of a consumer that another product or service fulfills (e.g. Pepsi and Coke or email and fax). We propose a two stage model where in the first stage firms choose their strategy which consists of the price they charge agents for their products and the quality of the products they offer. In the second stage, agents in the network decide what fraction of these two products to purchase. In essence this paper follows the same line of research as in [4] but our results and their interpretations are different which will be discussed in this paper. In our model each agent receives a utility irrespective of the consumptions of her peers and a utility which can be thought of as the payoff of a local coordination

\footnotetext{
${ }^{\dagger}$ Department of Electrical and Systems Engineering and GRASP Laboratory at University of Pennsylvania. arastoo@seas . upenn. edu and jadbabai@seas. upenn. edu. This research was supported by ONR MURI N000140810747 and AFOSR Complex Networks Program.
}

game played among her and her neighbors. There are diverse sets of products having this local coordination effect where consuming a common product has a higher payoff. Network goods and services (e.g., cell phones, fax machines and email accounts) are just a few examples of this kind of products where consumption of an agent has a positive impact on her peers' consumptions. This effect is often called positive network externality in the literature. In this paper, considering these types of products, we characterize the Nash equilibrium of agents' actions in the network and show how agents' consumptions in the Nash equilibrium depend on their Bonacich centrality which is a sociological measure of network influence [6]. We also show the effect of the quality and the price of products offered by firms on individual decisions. Conditioned on the consumption of agents, we find the Nash equilibrium of the pricing game played between firms. We show that even in the case of uniform price and similar firms, the price offered by firms depends on the network structure. We then consider row or column stochastic networks and show that the consumption of agents are equal, and the Nash equilibrium price is independent of the network structure. We analyze sensitivity of agents' consumption with respect to the price and quality of the products and show that agents are more sensitive with respect to the price. We finally compare the optimal duopoly price with the optimal price offered in a monopoly market.

Recently, the equilibria of network games with linear best response functions have been completely characterized in [6]. Furthermore, the issue of optimal pricing in a monopoly was recently characterized in [4]. These results, as well as ours, are based on the assumption of a quadratic payoff function for agents. This assumption is assumed in [5], [7] as well. In addition, the relationship between Bonacich centrality and Nash equilibrium has already been studied in Ballester et al. in [5]. Our goal is to apply these results in a duopoly pricing setup in social networks, where there are two stages of firms and agents' decisions, and as a result, can be thought of as a sequential game.

The rest of this paper is organized as follows: In section II, we introduce our model. In section III, we study the second stage game in which agents decide on their consumptions. In section IV, we study the pricing game played between two firms. In section V, we study the problem when the network is stochastic. Finally, in section VI, we conclude the paper.

\section{MODEL}

We consider a duopoly market in which two firms compete for selling two substitute products $a$ and $b$ to a set of agents $\mathcal{I}=\{1, \ldots, n\}$ embedded in a social network. The relationship among agents is represented by an interaction 
matrix $G$. The $i j$-th entry of $G$ denoted by $g_{i j}$ represents the weight that agent $i$ assigns to agent $j$. We assume that $g_{i j} \geq 0$ for all $i, j$ and $g_{i i}=0$ for all $i$. The problem consists of two stages. In the first stage, firms $a$ and $b$ set their prices, i.e. how much they want to charge agents for selling each unit of their products. They also determine the quality of their products. Let $p_{a}$ and $p_{b}$ be prices that firms $a$ and $b$ charge respectively. Denote by $q_{a}$ and $q_{b}$ the quality of the products. For instance, $q_{a}$ and $q_{b}$ could be related to the investment of firms on the technology of production in order to make their products more appealing and superior in the market. For agents, $q_{a}$ and $q_{b}$ can be interpreted as the payoff that any two agents $i$ and $j$ would achieve if they both purchase the same product. In other words, we can assume $q_{a}$ and $q_{b}$ are payoffs that are obtained from the following game

\begin{tabular}{|l|c|r|}
\hline & $x_{j}$ & $1-x_{j}$ \\
\hline$x_{i}$ & $q_{a}$ & 0 \\
\hline $1-x_{i}$ & 0 & $q_{b}$ \\
\hline
\end{tabular}

where agent $i$ 's action denoted by $0 \leq x_{i} \leq 1$ is the fraction that she chooses to purchase from the product $a$ and $0 \leq 1-x_{i} \leq 1$ represents the fraction that she decides to purchase from the product $b$. Note that the agents' actions (the amount to be purchased from each product) are normalized to sum up to one. Since agents benefit from the same action of their neighbors, this game could be thought of as a local coordination game. From the above table it follows easily that the payoff of agents $i$ and $j$ from their interaction is

$$
u_{i j}\left(x_{i}, x_{j}\right)=q_{a} x_{i} x_{j}+q_{b}\left(1-x_{i}\right)\left(1-x_{j}\right) .
$$

One could also interpret $x_{i}$ as the probability of purchasing the product $a$ and $1-x_{i}$ as the probability of purchasing the product $b$. With this interpretation it can be easily seen that $u_{i j}$ in equation (1) represents the expected payoff resulting from the interaction between agents $i$ and $j$. We also assume that each agent benefits from taking action $x_{i}$ irrespective of actions taken by her neighbors. The isolation payoff of purchasing product $a$ is represented by the following quadratic form function

$$
u_{i i}^{a}\left(x_{i}\right)=e_{i} x_{i}-f_{i} x_{i}^{2} .
$$

where both $e_{i}$ and $f_{i}$ are non-negative constants. Therefore, the total isolation payoff from two products is

$$
\begin{aligned}
& u_{i i}\left(x_{i}\right)=e_{i} x_{i}-f_{i} x_{i}^{2}+e_{i}\left(1-x_{i}\right)-f_{i}\left(1-x_{i}\right)^{2} \\
& =\left(e_{i}-f_{i}\right)+2 f_{i} x_{i}-2 f_{i} x_{i}^{2} .
\end{aligned}
$$

Note that the first term is constant and does not depend on the consumption of any product so we can neglect it. As a result, we can see that the isolation payoff of agents can be represented as the following form

$$
u_{i i}\left(x_{i}\right)=a_{i} x_{i}-a_{i} x_{i}^{2},
$$

where $a_{i}=2 f_{i}$. This means that the isolation utility of agent $i$ disappears when the agent purchases only one of the products $a$ or $b$, i.e. $x_{i}=0$ or $x_{i}=1$. Assuming quadratic form function for the isolation payoff not only makes the analysis more tractable, but also is a good second order approximation for the general class of concave payoff functions. As mentioned above, $p_{a}$ and $p_{b}$ are the prices of purchasing each unit of $a$ and $b$ for agents. Therefore, the total utility of agent $i$ from taking the action $x_{i}$ is

$$
\begin{aligned}
& U_{i}\left(x_{i}, \vec{x}_{-i}\right)=a_{i} x_{i}-a_{i} x_{i}^{2}+q_{a} \sum_{j=1}^{N} g_{i j} x_{i} x_{j} \\
& +q_{b} \sum_{j=1}^{N} g_{i j}\left(1-x_{i}\right)\left(1-x_{j}\right)-p_{a} x_{i}-p_{b}\left(1-x_{i}\right) .
\end{aligned}
$$

Here we assume firms $a$ and $b$ charge all agents with the same price. In the above equation $\vec{x}_{-i}$ denotes an action vector of all agents other than agent $i$. Also note that agent $i$ 's payoff from interacting with her neighbors is weighted by weights that she assigns to them. From equation (2) we can see that products $a$ and $b$ have a positive externality effect in the network, meaning that the usage level of an agent has a positive impact on the usage level of her neighbors. Therefore, it follows that $q_{a}$ and $q_{b}$ in addition to the payoff of a local coordination game, can be interpreted as coefficients of network externality of products $a$ and $b$ respectively. Notice that if we assume that $q_{a}=1, q_{b}=0$ and $p_{b}=0$ equation (2) turns into

$$
U_{i}\left(x_{i}, \vec{x}_{-i}\right)=a_{i} x_{i}-a_{i} x_{i}^{2}+\sum_{j=1}^{N} g_{i j} x_{i} x_{j}-p_{a} x_{i},
$$

which is the utility function of agents for a monopoly market studied in [4]. For firms we consider the following utility functions

$$
\begin{aligned}
& U_{a}\left(p_{a}, q_{a}, p_{b}, q_{b}\right)=\left(p_{a}-c_{a}\right) \sum_{i=1}^{N} x_{i} \\
& U_{b}\left(p_{b}, q_{b}, p_{a}, q_{a}\right)=\left(p_{b}-c_{b}\right) \sum_{i=1}^{N}\left(1-x_{i}\right),
\end{aligned}
$$

where $c_{a}$ and $c_{b}$ denotes the marginal cost of producing each unit of products $a$ and $b$ by firms $a$ and $b$ respectively and $x_{i}$ and $1-x_{i}$ denotes the amount of products $a$ and $b$ that agent $i$ purchases in the network.

\section{Second Stage Game}

In this section, we characterize the Nash equilibrium of agents' actions in the second stage and show that under an assumption mentioned later this Nash equilibrium is unique and related to the centrality of agents in the social network. Next we study the impact of firms' strategies on actions of agents in this Nash equilibrium. The approach used in this section is similar to the one used in [4] but the results that we find and their interpretations are different which will be discussed later in this section.

In order to find the Nash equilibrium, we first need to find the best responses of agents in (2). To find the best response of each agent, we take the derivative of her utility function in 
(2) with respect to her consumption level and set it to zero. Thus, we get

$$
x_{i}^{*}=\frac{1}{2}+\frac{p_{b}-p_{a}}{2 a_{i}}+\frac{q_{a}}{2 a_{i}} \sum_{j=1}^{N} g_{i j} x_{j}-\frac{q_{b}}{2 a_{i}} \sum_{j=1}^{N} g_{i j}\left(1-x_{j}\right) .
$$

Therefore, the best response of agent $i$ when other agents choose actions represented by the vector $\vec{x}_{-i}$ is

$$
\begin{array}{ll}
\beta_{i}\left(\vec{x}_{-i}\right)=x_{i}^{*} \quad \text { if } \quad & 0 \leq x_{i}^{*} \leq 1, \\
\beta_{i}\left(\vec{x}_{-i}\right)=0 \quad \text { if } \quad x_{i}^{*}<0, \\
\beta_{i}\left(\vec{x}_{-i}\right)=1 \quad \text { if } \quad x_{i}^{*}>1 .
\end{array}
$$

As it is expected the best response of an agent for consumption of a product is an increasing function of its quality and a decreasing function of its price. To express the result in matrix form, let us define the vector $\vec{a}=\left[a_{i}\right]$ and the matrix $A=\operatorname{diag}(\vec{a})$. Define sets $S=\left\{i: 0 \leq x_{i}^{*} \leq 1\right\}$, $S_{a}=\left\{i: x_{i}^{*}>1\right\}$ and $S_{b}=\left\{i: x_{i}^{*}<0\right\}$ and denote by $s, s_{a}$ and $s_{b}$ their cardinality respectively. Denote by $\vec{x}_{s}$ the vector of $x_{i}$ such that $i \in S$. Define the vector $\vec{a}_{s}$ and matrices $A_{s}, G_{s}$ similarly. Here we restrict our analysis to the set of agents whose best responses are in the feasible set $[0,1]$. Therefore, the equation (4) can be written as

$A_{s} \vec{x}_{s}=\frac{\vec{a}_{s}}{2}+\left(\frac{p_{b}-p_{a}}{2}\right) \mathbf{1}_{s}+\left(\frac{q_{a}}{2}\right) G_{s} \vec{x}_{s}-\left(\frac{q_{b}}{2}\right) G_{s}\left(\mathbf{1}_{s}-\vec{x}_{s}\right)$.

Rearranging terms in the above equation leads to

$$
\left(A_{s}-\left(\frac{q_{a}+q_{b}}{2}\right) G_{s}\right) \vec{x}_{s}=\frac{\vec{a}_{s}}{2}+\left(\frac{p_{b}-p_{a}}{2}\right) \mathbf{1}_{s}-\left(\frac{q_{b}}{2}\right) G_{s} \mathbf{1}_{s} .
$$

We now show that under an assumption the Nash equilibrium of the second stage game is unique.

Theorem 1: If for all $i \in \mathcal{I}, \frac{2 a_{i}}{q_{a}+q_{b}}>\sum_{j=1}^{N} g_{i j}$, then the second stage game $\mathcal{G}=\left\{\mathcal{I},\left\{U_{i}\right\}_{i \in \mathcal{I}},[0,1]_{i \in \mathcal{I}}\right\}$ has a unique Nash equilibrium. This Nash equilibrium is given by

$\vec{x}_{s}^{*}=\left(\frac{1}{2}\right)\left(A_{s}-\left(\frac{q_{a}+q_{b}}{2}\right) G_{s}\right)^{-1}\left(\vec{a}_{s}+\left(p_{b}-p_{a}\right) \mathbf{1}_{s}-q_{b} G_{s} \mathbf{1}_{s}\right)$, $x_{i}^{*}=0 \quad$ if $\quad \beta_{i}\left(\vec{x}_{-i}\right)=0$,

$x_{i}^{*}=1 \quad$ if $\quad \beta_{i}\left(\vec{x}_{-i}\right)=1$.

Proof: $\frac{2 a_{i}}{q_{a}+q_{b}}>\sum_{j=1}^{N} g_{i j}$ implies that $\frac{2 a_{i}}{q_{a}+q_{b}}>$ $\sum_{j \in S} g_{i j}$ for all $i \in S$. Therefore, $\left(\frac{q_{a}+q_{b}}{2}\right) A_{s}^{-1} G_{s} \overrightarrow{1}<$ $\overrightarrow{1}$. By Perron-Frobenius theorem, the spectral radius of $\left(\frac{q_{a}+q_{b}}{2}\right) A_{s}^{-1} G_{s}$ is strictly less than one. Hence, it follows that none of eigenvalues of $\left(I-\left(\frac{q_{a}+q_{b}}{2}\right) A_{s}^{-1} G_{s}\right)$ is zero and so this matrix is invertible. Consequently, the matrix $\left(A_{s}-\right.$ $\left.\left(\frac{q_{a}+q_{b}}{2}\right) G_{s}\right)$ is invertible as well. Therefore, we can multiply both sides of (5) from left side by $\left(A_{s}-\left(\frac{q_{a}+q_{b}}{2}\right) G_{s}\right)^{-1}$ and obtain the Nash equilibrium stated in the theorem. Invertibility of $\left(A_{s}-\left(\frac{q_{a}+q_{b}}{2}\right) G_{s}\right)$ also guarantees the uniqueness of the Nash equilibrium.

Corollary 1: In the special case that agents only differ in their network position, i.e., $a_{i}=a_{0}$ or $A_{s}=a_{0} I_{s}$, the Nash equilibrium for agents with $0 \leq x_{i}^{*} \leq 1$ is given by

$$
\begin{aligned}
& \vec{x}_{s}^{*}=\left(\frac{q_{b}}{q_{a}+q_{b}}\right) \mathbf{1}_{s}+\left(\frac{1}{2}+\frac{p_{b}-p_{a}}{2 a_{0}}-\frac{q_{b}}{q_{a}+q_{b}}\right) \\
& \left(I_{s}-\left(\frac{q_{a}+q_{b}}{2 a_{0}}\right) G_{s}\right)^{-1} \mathbf{1}_{s} .
\end{aligned}
$$

Proof: It can be easily shown that for all $G_{s}$ we have

$$
\left(I_{s}-\alpha G_{s}\right)^{-1} G_{s}=\frac{\left(I_{s}-\alpha G_{s}\right)^{-1}-I}{\alpha} .
$$

Therefore, assuming $A_{s}=a_{0} I_{s}$ and setting $\alpha=\frac{q_{a}+q_{b}}{2 a_{0}}$, equation (6) after simplification becomes

$$
\begin{aligned}
& \vec{x}_{s}^{*}=\left(\frac{q_{b}}{q_{a}+q_{b}}\right) \mathbf{1}_{s}+\left(\frac{1}{2}+\frac{p_{b}-p_{a}}{2 a_{0}}-\frac{q_{b}}{q_{a}+q_{b}}\right) \\
& \left(I_{s}-\left(\frac{q_{a}+q_{b}}{2 a_{0}}\right) G_{s}\right)^{-1} \mathbf{1}_{s} .
\end{aligned}
$$

We can easily see that if we set $q_{a}=1, q_{b}=0$ and $p_{b}=0$ in (6) we get

$$
\vec{x}_{s}^{*}=\left(\frac{1}{2}\right)\left(A_{s}-\frac{1}{2} G_{s}\right)^{-1}\left(\vec{a}_{s}-p_{a} \mathbf{1}_{s}\right),
$$

which is what was obtained in the monopoly pricing problem in [4]. Note that as opposed to the monopoly case, actions of agents in a duopoly market has a constant term which depends on the relative payoff of products. If we set $q_{b}=0$ we can see that this constant term disappears.

To obtain an alternative characterization of the Nash equilibrium in (6), we provide the definition of Bonacich Centrality.

Definition 1: Bonacich Centrality:

For a network with weighted adjacency matrix $G$ and scalar $\alpha$, the Bonacich centrality vector of parameter $\alpha$ is defined by $\kappa(G, \alpha)=(I-\alpha G)^{-1} \overrightarrow{1}$, if $(I-\alpha G)$ is invertible and non-negative.

Therefore, by this definition equation (7) becomes

$\vec{x}_{s}^{*}=\left(\frac{q_{b}}{q_{a}+q_{b}}\right) \mathbf{1}_{s}+\left(\frac{1}{2}+\frac{p_{b}-p_{a}}{2 a_{0}}-\frac{q_{b}}{q_{a}+q_{b}}\right) \kappa\left(G, \frac{q_{a}+q_{b}}{2 a_{0}}\right)$.

The first term in equation (8) is the amount that all agents purchase from the product $a$ in the Nash equilibrium irrespective of the network structure. In other words, this term, i.e. $x^{c}=\frac{q_{b}}{q_{a}+q_{b}}=\frac{1}{\frac{q_{a}}{q_{b}}+1}$, can be thought of as the offset of agents' actions in the Nash equilibrium. The second term which is proportional to the agents' centrality shows how much agents are inclined to purchase the product $a$ or $b$ from their offset actions. In equation (8) we can see that the parameter of centrality is only function of the quality of products, i.e. $q_{a}$ and $q_{b}$. However, the coefficient which amplifies the centrality effect is a function of both the quality and the price, i.e. $p_{a}, p_{b}, q_{a}$ and $q_{b}$. As we expected and can be easily seen from equation (8), $\vec{x}_{s}^{*}$ is a decreasing (increasing) function of $p_{a}\left(p_{b}\right)$. This means that increasing the price of products by firms leads to less consumption of that product by agents. On the other hand, the consumption at the equilibrium would not change if both firms change their prices in the same amount. Knowing this fact might potentially result in collusion between firms in order to increase their price and make more profit. Also, we can see 
that the offset of actions is a function of the ratio of products' payoffs, i.e. $\frac{q_{a}}{q_{b}}$. However, contrary to our expectation, $x^{c}$ is a decreasing function of $\frac{q_{a}}{q_{b}}$, meaning that the agents purchase less of product $a$ when $\frac{q_{a}}{q_{b}}$ is greater or when the product $a$ is more attractive to them. The reason for this contrary is that we have not considered the effect of the second term yet. In section IV we will see that as we expected the consumption of a product in Nash equilibrium is an increasing function of its payoff and a decreasing function of the other product's payoff.

In the next section, we study the game between firms when agents take their actions in the Nash equilibrium as it was described in this section.

\section{First Stage Game}

In this section we obtain utility functions of firms resulting from actions of agents in the Nash equilibrium. We find the best response functions of firms from their utility functions. Using best response functions, we finally characterize prices of firms in the Nash equilibrium of the game played between them. Finally, we conclude this section with an analysis of our results.

Theorem 2: Conditioned on the actions of agents in the Nash equilibrium of the second stage game in (6) and when $s_{a}=s_{b}=0$ (restricting the analysis and the network to those agents with feasible best responses), the Nash equilibrium of the game between firms with utility functions defined in (3) is given by

$$
\begin{aligned}
& p_{a}^{*}=\frac{2 c_{a}+c_{b}}{3}+\left(\frac{q_{a}-q_{b}}{3\left(q_{a}+q_{b}\right)}\right) \frac{\mathbf{1}_{s}^{T}\left(A_{s}-\left(\frac{q_{a}+q_{b}}{2}\right) G_{s}\right)^{-1} \vec{a}_{s}}{\mathbf{1}_{s}^{T}\left(A_{s}-\left(\frac{q_{a}+q_{b}}{2}\right) G_{s}\right)^{-1} \mathbf{1}_{s}} \\
& +\left(\frac{2\left(q_{a}+2 q_{b}\right)}{3\left(q_{a}+q_{b}\right)}\right) \frac{\mathbf{1}_{s}^{T} \mathbf{1}_{s}}{\mathbf{1}_{s}^{T}\left(A_{s}-\left(\frac{q_{a}+q_{b}}{2}\right) G_{s}\right)^{-1} \mathbf{1}_{s}}, \\
& p_{b}^{*}=\frac{2 c_{b}+c_{a}}{3}+\left(\frac{q_{b}-q_{a}}{3\left(q_{a}+q_{b}\right)}\right) \frac{\mathbf{1}_{s}^{T}\left(A_{s}-\left(\frac{q_{a}+q_{b}}{2}\right) G_{s}\right)^{-1} \vec{a}_{s}}{\mathbf{1}_{s}^{T}\left(A_{s}-\left(\frac{q_{a}+q_{b}}{2}\right) G_{s}\right)^{-1} \mathbf{1}_{s}} \\
& +\left(\frac{2\left(q_{b}+2 q_{a}\right)}{3\left(q_{a}+q_{b}\right)}\right) \frac{\mathbf{1}_{s}^{T} \mathbf{1}_{s}}{\mathbf{1}_{s}^{T}\left(A_{s}-\left(\frac{q_{a}+q_{b}}{2}\right) G_{s}\right)^{-1} \mathbf{1}_{s}} .
\end{aligned}
$$

Proof: If we plug actions of agents in the Nash equilibrium in (6) into the utility functions of firms in equation (3) we get the following utility functions for firms

$$
\begin{aligned}
& U_{a}\left(p_{a}, q_{a}, p_{b}, q_{b}\right)=\left(p_{a}-c_{a}\right) \mathbf{1}^{T} \vec{x}^{*}=\left(p_{a}-c_{a}\right) \mathbf{1}_{s}^{T}\left\{( \frac { 1 } { 2 } ) \left(A_{s}-\right.\right. \\
& \left.\left.\left(\frac{q_{a}+q_{b}}{2}\right) G_{s}\right)^{-1}\left(\vec{a}_{s}+\left(p_{b}-p_{a}\right) \mathbf{1}_{s}-q_{b} G_{s} \mathbf{1}_{s}\right)\right\}+\left(p_{a}-c_{a}\right) s_{a}
\end{aligned}
$$

$U_{b}\left(p_{b}, q_{b}, p_{a}, q_{a}\right)=\left(p_{b}-c_{b}\right) \mathbf{1}_{s}^{T}\left\{\mathbf{1}_{s}-\left(\frac{1}{2}\right)\left(A_{s}-\left(\frac{q_{a}+q_{b}}{2}\right) G_{s}\right)^{-1}\right.$ $\left(\vec{a}_{s}+\left(p_{b}-p_{a}\right) \mathbf{1}_{s}-q_{b} G_{s} \mathbf{1}_{s}\right\}+\left(p_{b}-c_{b}\right) s_{b}$.

In order to find the optimal price offered by firms, we take the derivative of their utility functions with respect to their prices and set it to zero. This is in fact the best response of a firm conditioned on the price of the other firm. After simplification and using the fact that $A_{s} \mathbf{1}_{s}=\vec{a}_{s}$ and

$$
\left(A_{s}-\alpha G_{s}\right)^{-1} G=\frac{\left(A_{s}-\alpha G_{s}\right)^{-1} A_{s}-I}{\alpha},
$$

for best responses of firms we obtain

$$
\begin{aligned}
& \beta_{a}\left(p_{b}\right)=\frac{p_{b}+c_{a}}{2}+\left(\frac{q_{a}-q_{b}}{2\left(q_{a}+q_{b}\right)}\right) \frac{\mathbf{1}_{s}^{T}\left(A_{s}-\left(\frac{q_{a}+q_{b}}{2}\right) G_{s}\right)^{-1} \vec{a}_{s}}{\mathbf{1}_{s}^{T}\left(A_{s}-\left(\frac{q_{a}+q_{b}}{2}\right) G_{s}\right)^{-1} \mathbf{1}_{s}} \\
& +\frac{\left(\frac{q_{b}}{q_{a}+q_{b}}\right) s+s_{a}}{\mathbf{1}_{s}^{T}\left(A_{s}-\left(\frac{q_{a}+q_{b}}{2}\right) G_{s}\right)^{-1} \mathbf{1}_{s}}, \\
& \beta_{b}\left(p_{a}\right)=\frac{p_{a}+c_{b}}{2}+\left(\frac{q_{b}-q_{a}}{2\left(q_{a}+q_{b}\right)}\right) \frac{\mathbf{1}_{s}^{T}\left(A_{s}-\left(\frac{q_{a}+q_{b}}{2}\right) G_{s}\right)^{-1} \vec{a}_{s}}{\mathbf{1}_{s}^{T}\left(A_{s}-\left(\frac{q_{a}+q_{b}}{2}\right) G_{s}\right)^{-1} \mathbf{1}_{s}} \\
& +\frac{\left(\frac{q_{a}}{q_{a}+q_{b}}\right) s+s_{b}}{\mathbf{1}_{s}^{T}\left(A_{s}-\left(\frac{q_{a}+q_{b}}{2}\right) G_{s}\right)^{-1} \mathbf{1}_{s}} .
\end{aligned}
$$

Notice that if in the above equation we set $q_{a}=1, q_{b}=0$, $p_{b}=0$ and $s_{a}=0$, we get

$$
\beta_{a}\left(p_{b}\right)=\frac{c_{a}}{2}+\left(\frac{1}{2}\right) \frac{\mathbf{1}_{s}^{T}\left(A_{s}-\left(\frac{1}{2}\right) G_{s}\right)^{-1} \vec{a}_{s}}{\mathbf{1}_{s}^{T}\left(A_{s}-\left(\frac{1}{2}\right) G_{s}\right)^{-1} \mathbf{1}_{s}},
$$

which is the optimal price charged by a monopolist obtained in [4]. Now in order to find the Nash equilibrium of the game between firms, we plug the best response of each firm into the other firm's best response and solve the resulting equation. After simplification we get the following Nash equilibrium in the first stage game

$$
\begin{aligned}
& p_{a}^{*}=\frac{2 c_{a}+c_{b}}{3}+\left(\frac{q_{b}-q_{a}}{3\left(q_{a}+q_{b}\right)}\right) \frac{\mathbf{1}_{s}^{T}\left(A_{s}-\left(\frac{q_{a}+q_{b}}{2}\right) G_{s}\right)^{-1} \vec{a}_{s}}{\mathbf{1}_{s}^{T}\left(A_{s}-\left(\frac{q_{a}+q_{b}}{2}\right) G_{s}\right)^{-1} \mathbf{1}_{s}} \\
& +\frac{\left(\frac{2 s}{3}\right)\left(\frac{q_{b}}{q_{a}+q_{b}}+1+\frac{2 s_{a}}{s}+\frac{s_{b}}{s}\right)}{\mathbf{1}_{s}^{T}\left(A_{s}-\left(\frac{q_{a}+q_{b}}{2}\right) G_{s}\right)^{-1} \mathbf{1}_{s}}, \\
& p_{b}^{*}=\frac{2 c_{b}+c_{a}}{3}+\left(\frac{q_{a}-q_{b}}{3\left(q_{a}+q_{b}\right)}\right) \frac{\mathbf{1}_{s}^{T}\left(A_{s}-\left(\frac{q_{a}+q_{b}}{2}\right) G_{s}\right)^{-1} \vec{a}_{s}}{\mathbf{1}_{s}^{T}\left(A_{s}-\left(\frac{q_{a}+q_{b}}{2}\right) G_{s}\right)^{-1} \mathbf{1}_{s}} \\
& +\frac{\left(\frac{2 s}{3}\right)\left(\frac{q_{a}}{q_{a}+q_{b}}+1+\frac{2 s_{b}}{s}+\frac{s_{a}}{s}\right)}{\mathbf{1}_{s}^{T}\left(A_{s}-\left(\frac{q_{a}+q_{b}}{2}\right) G_{s}\right)^{-1} \mathbf{1}_{s}} .
\end{aligned}
$$

If we let $s_{a}=s_{b}=0$ and $s=\mathbf{1}_{s}^{T} \mathbf{1}_{s}$ we get the result in theorem 2 .

Theorem 2 implicitly shows the effect of the cost and the quality of a firm's product on her Nash equilibrium price. However, in order to see this effect more clearly we need to make the model simpler. We investigate these effects further in section IV.

Corollary 2: When agents only differ in their network position, i.e., $a_{i}=a_{0}$ for all $i \in \mathcal{I}$ and $s_{a}=s_{b}=0$, the Nash equilibrium of the game between firms is given by

$$
\begin{aligned}
& p_{a}^{*}=\frac{2 c_{a}+c_{b}}{3}+\frac{a_{0}\left(q_{a}-q_{b}\right)}{3\left(q_{a}+q_{b}\right)} \\
& +\left(\frac{2 a_{0}\left(q_{a}+2 q_{b}\right)}{3\left(q_{a}+q_{b}\right)}\right) \frac{\mathbf{1}_{s}^{T} \mathbf{1}_{s}}{\mathbf{1}_{s}^{T}\left(I_{s}-\alpha G_{s}\right)^{-1} \mathbf{1}_{s}}, \\
& p_{b}^{*}=\frac{2 c_{b}+c_{a}}{3}+\frac{a_{0}\left(q_{b}-q_{a}\right)}{3\left(q_{a}+q_{b}\right)} \\
& +\left(\frac{2 a_{0}\left(q_{a}+2 q_{b}\right)}{3\left(q_{a}+q_{b}\right)}\right) \frac{\mathbf{1}_{s}^{T} \mathbf{1}_{s}}{\mathbf{1}_{s}^{T}\left(I_{s}-\alpha G_{s}\right)^{-1} \mathbf{1}_{s}} .
\end{aligned}
$$


where $\alpha=\frac{q_{a}+q_{b}}{2 a_{0}}$.

Proof: From equation (10) and after setting $A_{s}=a_{0} I_{s}$ and $\vec{a}_{s}=a_{0} \mathbf{1}_{s}$ we get

$p_{a}^{*}=\frac{\left(\frac{2 a_{0} s}{3}\right)\left(\frac{q_{b}}{q_{a}+q_{b}}+\frac{2 s_{a}}{s}+\frac{s_{b}}{s}+1\right)}{\mathbf{1}_{s}^{T}\left(I_{s}-\alpha G_{s}\right)^{-1} \mathbf{1}_{s}}+\frac{a_{0}\left(q_{a}-q_{b}\right)}{3\left(q_{a}+q_{b}\right)}+\frac{2 c_{a}+c_{b}}{3}$,

$p_{b}^{*}=\frac{\left(\frac{2 a_{0} s}{3}\right)\left(\frac{q_{a}}{q_{a}+q_{b}}+\frac{2 s_{b}}{s}+\frac{s_{a}}{s}+1\right)}{\mathbf{1}_{s}^{T}\left(I_{s}-\alpha G_{s}\right)^{-1} \mathbf{1}_{s}}+\frac{a_{0}\left(q_{b}-q_{a}\right)}{3\left(q_{a}+q_{b}\right)}+\frac{2 c_{b}+c_{a}}{3}$.

Now if we let $s_{a}=s_{b}=0$ and $s=\mathbf{1}_{s}^{T} \mathbf{1}_{s}$ we get the result in corollary 2 .

If we consider the case where both firms have the same cost and quality for their products, i.e. $q_{a}=q_{b}=q$ and $c_{a}=c_{b}=c$, and also assuming $s_{a}=s_{b}=0$, then the Nash equilibrium becomes

$$
p^{*}=c+\frac{a_{0} \mathbf{1}_{s}^{T} \mathbf{1}_{s}}{\mathbf{1}_{s}^{T}\left(I_{s}-\alpha G_{s}\right)^{-1} \mathbf{1}_{s}} .
$$

This result indicates that even when all agents are homogenous and firms are similar, the Nash equilibrium price depends on the network structure. This result is different from the monopoly market where the optimal price is independent of the network structure and is given by

$$
p^{*}=\frac{c+a_{0}}{2},
$$

when agents are homogenous. In the next section, we study the Nash equilibrium of the first and second stage games when the network is row or column stochastic and give an analysis of our results.

\section{Nash Equilibrium in Stochastic Networks}

In this section we study the Nash equilibrium of the second and first stage games when agents only differ in their network position, i.e., $a_{i}=a_{0}$ for all $i \in \mathcal{I}$ and the weighted adjacency matrix of the network is normalized to a row or column stochastic matrix. If matrix $G_{s}$ is row (column) stochastic, then it means that its rows (columns) sum up to one. In other words, it has a right (left) eigenvector of $\mathbf{1}_{s}$ corresponding to its eigenvalue of $\lambda_{1}=1$. Therefore, we have one of these two cases

$$
\begin{aligned}
& G_{s} \mathbf{1}_{s}=\mathbf{1}_{s} \text { if } G_{s} \text { is row stochatsic, } \\
& \mathbf{1}_{s}^{T} G_{s}=\mathbf{1}_{s}^{T} \text { if } G_{s} \text { is column stochatsic. }
\end{aligned}
$$

In the next corollary we describe actions of agents in the Nash equilibrium when the weighted adjacency matrix of the network is normalized to a row stochastic matrix and compare its sensitivity with respect to the price and the payoff a product.

Corollary 3: If the weight adjacency matrix of the network $G$ is normalized to a row stochastic matrix and agents only differ in their network position, i.e., $a_{i}=a_{0}$ for all $i \in \mathcal{I}$, then in the Nash equilibrium the consumptions of all agents are equal and independent of the network structure and is given by

$$
\vec{x}_{s}^{*}=\left(\frac{a_{0}+p_{b}-p_{a}-q_{b}}{2 a_{0}-\left(q_{a}+q_{b}\right)}\right) \mathbf{1}_{s} .
$$

Moreover, agents consumption is more sensitive with respect to the price than to the quality. In other words,

$$
\begin{aligned}
& \left\|\frac{\partial x^{*}}{\partial q_{a}}\right\| \leq\left\|\frac{\partial x^{*}}{\partial p_{a}}\right\|, \\
& \left\|\frac{\partial\left(1-x^{*}\right)}{\partial q_{b}}\right\| \leq\left\|\frac{\partial\left(1-x^{*}\right)}{\partial p_{b}}\right\| .
\end{aligned}
$$

Proof: First note that from the definition of centrality we have

$$
\left(I-\alpha G_{s}\right)^{-1}=\Sigma_{k=0}^{\infty}\left(\alpha G_{s}\right)^{k} .
$$

It can be easily seen that for any power of row (column) stochastic matrix $G_{s}$, the vector $\mathbf{1}_{s}$ is still its right (left) eigenvector corresponding to the eigenvalue of $\lambda_{1}=1$. Therefore, from equation (12) we have

$$
\begin{aligned}
& \left(I-\alpha G_{s}\right)^{-1} \mathbf{1}_{s}=\Sigma_{k=0}^{\infty}\left(\alpha^{k}\right) G_{s}^{k} \mathbf{1}_{s} \\
& =\Sigma_{k=0}^{\infty}\left(\alpha^{k}\right) \mathbf{1}_{s}=\left(\frac{1}{1-\alpha}\right) \mathbf{1}_{s} .
\end{aligned}
$$

Therefore, setting $\alpha=\frac{q_{a}+q_{b}}{2 a_{0}}$, rearranging terms in equation (8) and simplifying them, we obtain the following actions vector for a row stochastic network

$$
\vec{x}_{s}^{*}=\left(\frac{a_{0}+p_{b}-p_{a}-q_{b}}{2 a_{0}-\left(q_{a}+q_{b}\right)}\right) \mathbf{1}_{s} .
$$

From this equation and the assumption of $2 a_{0}>q_{a}+q_{b}$ in section II, it can be seen that as we had expected $\vec{x}_{s}^{*}$ is an increasing (decreasing) function of $q_{a}\left(q_{b}\right)$ meaning that the quality (payoff) of a product leads to more consumption of that product. Also, $\vec{x}_{s}^{*}$ is a decreasing (increasing) function of $p_{a}\left(p_{b}\right)$ meaning that if a firm charges more for her product, people consume less. More precisely, we can see that for firm $a$ we have

$$
\begin{aligned}
& \frac{\partial x^{*}}{\partial p_{a}}=\frac{-1}{2 a_{0}-\left(q_{a}+q_{b}\right)} \\
& \frac{\partial x^{*}}{\partial q_{a}}=\frac{a_{0}+p_{b}-p_{a}-q_{b}}{\left(2 a_{0}-\left(q_{a}+q_{b}\right)\right)^{2}}
\end{aligned}
$$

Since $x^{*} \leq 1$, it follows that $\left\|\frac{\partial x^{*}}{\partial q_{a}}\right\| \leq\left\|\frac{\partial x^{*}}{\partial p_{a}}\right\|$. Similarly, we can see that

$$
\begin{aligned}
& \frac{\partial\left(1-x^{*}\right)}{\partial p_{a}}=\frac{-1}{2 a_{0}-\left(q_{a}+q_{b}\right)}, \\
& \frac{\partial\left(1-x^{*}\right)}{\partial q_{a}}=\frac{a_{0}+p_{a}-p_{b}-q_{a}}{\left(2 a_{0}-\left(q_{a}+q_{b}\right)\right)^{2}} .
\end{aligned}
$$

Hence, $\left\|\frac{\partial\left(1-x^{*}\right)}{\partial q_{b}}\right\| \leq\left\|\frac{\partial\left(1-x^{*}\right)}{\partial p_{b}}\right\|$. Therefore, in the Nash equilibrium the consumption of an agent is more sensitive with respect to the price than to the quality (payoff).

Note that from equation (13) we can also see that agents are more sensitive with respect to the price when the quality of a product is higher. In other words,

$$
\frac{\partial^{2}\left(x^{*}\right)}{\partial q_{a} \partial p_{a}}<0 \quad \frac{\partial^{2}\left(1-x^{*}\right)}{\partial q_{b} \partial p_{b}}<0 .
$$

Corollary 4: If the weighted adjacency matrix of a network is normalized to a row or column stochastic matrix and agents only differ in their network position, i.e. $a_{i}=a_{0}$ for all $i \in \mathcal{I}$, and $s_{a}=s_{b}=0$, then the price in the Nash 
equilibrium of the game between firms is independent of the network structure and is given by

$$
\begin{aligned}
& p_{a}^{*}=\frac{3 a_{0}+2 c_{a}+c_{b}-q_{a}-2 q_{b}}{3}, \\
& p_{b}^{*}=\frac{3 a_{0}+2 c_{b}+c_{a}-q_{b}-2 q_{a}}{3} .
\end{aligned}
$$

Proof: From the fact that for any row (column) stochastic graph $G$, the vector $\mathbf{1}_{s}$ is still its right (left) eigenvector corresponding to the eigenvalue of $\lambda_{1}=1$ of $G_{s}^{k}$ we get

$$
\begin{aligned}
& \mathbf{1}_{s}^{T}\left(I-\alpha G_{s}\right)^{-1} \mathbf{1}_{s}=\Sigma_{k=0}^{\infty}\left(\alpha^{k}\right) \mathbf{1}_{s}^{T} G_{s}^{k} \mathbf{1}_{s} \\
& =\Sigma_{k=0}^{\infty}\left(\alpha^{k}\right) \mathbf{1}_{s}^{T} \mathbf{1}_{s}=s \Sigma_{k=0}^{\infty} \alpha^{k}=s \frac{1}{1-\alpha} .
\end{aligned}
$$

Therefore, using this fact, setting $\alpha=\frac{q_{a}+q_{b}}{2 a_{0}}$ and $s_{a}=s_{b}=$ 0 along with equation (9) we get following best responses for firms

$$
\begin{aligned}
& \beta_{a}\left(p_{b}\right)=\frac{a_{0}+c_{a}+p_{b}-q_{b}}{2}, \\
& \beta_{b}\left(p_{a}\right)=\frac{a_{0}+c_{b}+p_{a}-q_{a}}{2} .
\end{aligned}
$$

Solving the above equation for prices, we get the result in Corollary 4.

First notice that if two firms are similar, i.e. $c_{a}=c_{b}$ and $q_{a}=q_{b}$, the Nash equilibrium in corollary 4 becomes

$$
p^{*}=a_{0}+c-q .
$$

Also note that equation (14) implies that increasing a firm's price results in increasing her opponent's best response price. This is because of the fact that by increasing a firm's price agents in the market become more inclined towards the other firm. As a result, the other firm can take advantage of this opportunity and increase her price too. Another point here is that a firm's best response price decreases if her opponent increases her product's quality. This is clear due to the competitive nature of the market, since when her opponent increases her product's quality, the firm has to decrease her price so that she does not lose her costumers. Also notice that the price of a firm in Nash equilibrium in corollary 4 is not only an increasing function of her cost, but also an increasing function of her opponent's cost. This could be due to the fact that if her opponent's cost increases, then her opponent has to increase her price to cover her cost and as it was explained this yields to the increasing of the firm's best response price. Moreover, corollary 4 states that in the Nash equilibrium increasing a firm's product quality results in decreasing her price. This might be counter intuitive at the first glance but can be explained in the following way. If a firm increases her product's quality, as can be seen from equation (14) her opponent's best response price decreases. This in turn leads to the decreasing of the firm's best response price. Also notice that the optimal price of a monopolist in this case is given by

$$
p_{m}^{*}=\frac{a_{0}+c}{2} .
$$

Comparing this with the best responses of firms in equation (14), it follows that depending on the sign of $p-q$ of a firm's opponent, the best response price of the firm in duopoly market can be greater, equal or less than her optimal price in a monopoly market. In other words, if her opponent charges more (less) price than the quality that she provides for her product, the best response price of the firm would be higher (lower) than as if she was in a monopoly market.

\section{CONCLUSION}

We studied a duopoly pricing problem of competing firms in social networks. Two firms selling substitute products in a social network decide on the uniform price that they charge agents for their products and the quality of the products they offer. For agents, the quality is the payoff of a local coordination game played among them in the network. This payoff can also be interpreted as the positive externality effect of a product in the network. After firms make their decisions, agents in the network decide what fraction of these two products to purchase. Similar to results of [6] and [4] we showed that agents consumption in the Nash equilibrium consists of a constant term and a term which depends on their Bonacich centrality in the network. We then characterized the Nash equilibrium of two firms and showed that even when firms are similar and offer a single uniform price for all agents, their price in the Nash equilibrium depends on the structure of the network, which is different from the monopoly case. Next we considered the case when the adjacency matrix of the network is normalized. We showed that in this case, consumptions of all agents are equal and Nash equilibrium price is independent of the network structure. We then analyzed the sensitivity of an agent's consumption with respect to the price and the quality of a product and showed that an agent is more sensitive with respect to the price. We finally showed that in this case, depending on a firm's opponent's price and quality, the optimal price of the firm can be higher, equal or less than the monopoly's optimal price. Directions for future research could include studying distinctive prices for agents and also the case that there are more than two products in the market and the relationship among firms also is represented by a network.

\section{REFERENCES}

[1] L. Feick and L. Price, "The market maven: A diffuser of marketplace information," The Journal of Marketing, pp. 83-97, 1987.

[2] P. Reingen, B. Foster, J. Brown, and S. Seidman, "Brand congruence in interpersonal relations: A social network analysis," Journal of Consumer Research, pp. 771-783, 1984.

[3] D. Godes and D. Mayzlin, "Using online conversations to study wordof-mouth communication," Marketing Science, pp. 545-560, 2004.

[4] O. Candogan, K. Bimpikis, and A. Ozdaglar, "Optimal pricing in networks with externalities," Arxiv preprint arXiv:1101.5617, 2011.

[5] C. Ballester, A. Calvó-Armengol, and Y. Zenou, "Who's who in networks. wanted: the key player," Econometrica, vol. 74, no. 5, pp. $1403-$ 1417, 2006.

[6] Y. Bramoullé and R. Kranton, and M. DAmours, "Strategic interaction and networks,", 2009.

[7] J. Corbo, A. Calvó-Armengol, and D. Parkes, "The importance of network topology in local contribution games," in Proceedings of the 3rd international conference on Internet and network economics, pp. 388-395, Springer-Verlag, 2007.

[8] A. Galeotti and S. Goyal, "Influencing the influencers: a theory of strategic diffusion," The RAND Journal of Economics, vol. 40, no. 3, pp. 509-532, 2009. 